\title{
Immunogold studies of monomeric elements from the globular domain (NC1) of type IV collagen in renal basement membranes during experimental diabetes in the rat
}

\author{
M. Desjardins ${ }^{1}$, F. Gros ${ }^{1,2}$, J. Wieslander ${ }^{3}$, M.C. Gubler ${ }^{2}$ and M.Bendayan ${ }^{1}$ \\ Département d'Anatomie, Faculté de Médecine, Université de Montréal, Montréal, Québec, Canada \\ 2 INSERM U.192, Hôpital Necker pour les Enfants Malades, Paris, France \\ ${ }^{3}$ Biocarb AB, Lund, Sweden
}

\begin{abstract}
Summary. The protein A-gold immunocytochemical technique was applied to reveal the monomeric elements M1, $\mathrm{M} 2 *$ and M3 from the non-collagenous globular domain (NC1) of type IV collagen over various renal basement membranes from control and long-term streptozotocin-induced diabetic rats. This study includes the basement membranes of the proximal tubule, the Bowman's capsule and the glomerulus as well as the extracellular matrix of the mesangium. The labellings obtained were confined to basement membrane material. The quantitative analysis demonstrated changes in labelling intensities and distribution between tissues from normal and diabetic animals. Increased labelling intensities were observed for $\mathrm{M} 1$ and $\mathrm{M} 2 *$ monomers in all the basement membranes studied except for the mesangial matrix which remained unchanged. In addition, the labelling for M1 monomers, present on the endothelial side of the glomerular basement membrane of control animals, was found to be dis-
\end{abstract}

tributed throughout the entire thickness of the basement membrane of diabetic animals. In contrast, neither the intensity of the labelling, nor the distribution of M3 monomers were altered in diabetic animals. Since M1 monomers are markers of the $\alpha 1$ (IV) and $\alpha 2$ (IV) chains of type IV collagen while M2* and M3 mark $\alpha 3$ (IV) and $\alpha 4$ (IV) chains respectively, the present results demonstrate changes in the nature of the collagenous elements of basement membranes during diabetes. Furthermore, the results indicate that the $\alpha 3$ (IV) and the $\alpha 4$ (IV) chains are not necessarily present in the same molecule. The modifications of the collagenous elements of the basement membranes during diabetes must alter the structural characteristics of these matrices which in turn might influence their functional properties.

Key words: Type IV collagen, basement membranes, immunocytochemistry, kidney, diabetes.
Capillary basement membranes are the sites of major morphological and biochemical alterations during diabetes. In the kidney, spreading of the mesangial matrix and thickening of the glomerular basement membrane are the most striking morphological alterations [1-4] which occur concomitant to a progressive impairment of the functional properties with loss of the glomerular permselectivity and increase proteinuria [5-8]. Alterations in the composition of the glomerular basement membrane in diabetic patients as well as in experimentally induced diabetic rats have also been reported. Although challenged, they mainly consist in alterations of the amino acids and glycoconjugates composition [9-15], as well as in modifications of the content of theirmacromolecular components [16-19], such as type IV collagen, laminin, and proteoglycans. In addition to the biochemical increase of type IV collagen, its distribution within the glomerular basement membrane was found to be modified [20]. These modifications are believed to influence the molecular organization of the glomerular basement membrane which in turn must alter its physiological properties.
Type IV collagen molecules have been shown to interact among themselves to form the basic framework of basement membranes [21-27]. Recent data demonstrated that the non-collagenous domain ( $\mathrm{NC1}$ ) from two ad jacent type IV collagen molecules, extracted from bovine glomerular basement membrane, is made of four types of monomeric structures namely M1a, M1b, M2* and M3 $[28,29]$. Biochemical analysis showed that M1a and M1b monomers are associated to the $\alpha 1$ (IV) and $\alpha 2$ (IV) chains of type IV collagen, while $\mathrm{M} 2 *$ and $\mathrm{M} 3$ are considered as part of two new chains designated $\alpha 3$ (IV) and $\alpha 4$ (IV) respectively [29]. These monomers are not evenly distributed within the normal renal basement membranes as demonstrated by immunofluorescence $[30,31]$ and the high resolution immunogold technique [32] confirming the concept of basement membrane heterogeneity [33]. In diabetic patients, we have previously shown by immunofluorescence that mesangial sclerosis is associated with accumulation of solely M1 monomers, whereas both M1 and $\mathrm{M} 2 *$ monomers participate in the thickening of the glomerular basement membrane [34]. 
The aim of the present study was to further investigate, at the ultrastructural level, changes in the distribution of M1, M2* and M3 monomers in renal basement membranes during diabetes. To do so, the protein A-gold technique was applied to localize the antigenic sites of these peptides in renal tissues from control and long-term streptozotocin-induced diabetic rats. The high resolution of the labelling achieved by the use of the gold marker has further enabled morphometrical analysis. The results revealed changes in the pattern of labelling of the monomers indicating alterations of type IV collagen chains in basement membranes during diabetes.

\section{Materials and methods}

An experimental chronic hyperglycaemic state was induced in $100 \mathrm{~g}$ Sprague-Dawley male rats by an intraperitoneal injection of streptozotocin $(70 \mathrm{mg} / \mathrm{kg}$ body weight, in citrate buffer $10 \mathrm{mmol} / \mathrm{l}$, $\mathrm{pH} 4.5$ ). Control animals only received an injection of the citrate buffer. The animals of the experimental group remained hyperglycaemic throughout the entire length of the experiment, as demonstrated by the frequent glycosuria and occasional glycaemia measurements made using the Multistix and Dextrostix reagent strips (Miles, Ames, Ontario, Canada), while those injected with the citrate buffer remained normoglycaemic. The animals were fed ad libitum with standard rat chow and had free access to water. After 12 months of hyperglycaemia the rats were killed by decapitation and blood samples were taken for the determination of glucose and insulin levels. These were performed respectively by the glucose oxidase method and radioimmunoassay. Small fragments of cortical rat kidney were immediately fixed by immersion in periodate-lysine, paraformaldehyde $4 \%$ solution [35] for $2 \mathrm{~h}$ at $4^{\circ} \mathrm{C}$. The tissue fragments were then rinsed in $0.1 \mathrm{~mol} / 1$ phosphate buffer, dehydrated in methanol and embedded in Lowicryl $\mathrm{K} 4 \mathrm{M}$ at $-20^{\circ} \mathrm{C}$ as described previously [36]. Thin sections were cut, mounted on nickel grids coated with Parlodion and carbon films and processed for the cytochemical labelling.

Specific polyclonal antibodies raised in rabbits immunized with $\mathrm{M} 1, \mathrm{M} 2 *$ and M3 monomers of the globular (NC1) domain of type IV collagen extracted from bovine glomerular basement membrane were used [37]. These antibodies have been shown to crossreact with human and rat antigens by immunochemical and cytochemical approaches $[32,34]$. On the other hand, amino-acid sequences of the $\mathrm{NC1}$ peptides from various species were found to be highly homologous [38, 39]. M1 and M2* antibodies (IgG fractions) and a complete $\mathrm{M} 3$ antiserum $[28,40]$ were combined with the protein A-gold technique [36] to reveal the corresponding antigenic sites on the renal tissues as described previously [32].

In brief, tissue sections were incubated for $5 \mathrm{~min}$ on a drop of $0.01 \mathrm{~mol} / /$ phosphate buffered saline, $\mathrm{pH} 7.3$ (PBS) containing $1 \%$ ovalbumin, transferred to a drop of the antibody and incubated at room temperature for $90 \mathrm{~min}$. The grids were then rinsed with PBS, incubated with the PBS ovalbumin solution for $5 \mathrm{~min}$ and then with the protein A-gold for $30 \mathrm{~min}$ at room temperature. They were then washed with PBS, rinsed with distilled water and dried. Staining with uranyl acetate and lead citrate was performed prior to examination with a Siemens 101 electron microscope. For each antigen, the labelling procedure was performed simultaneously on tissue sections from all the animals. Protein A-gold complex was prepared with $15 \mathrm{~nm}$ gold particles as described previously [36]. The specificity of the antibodies was assessed through immunoblot experiments [28, 40]. In addition, experiments were performed in order to control the specificity of the labellings; these included incubation of the tissue sections with a normal serum prior to the protein A-gold complex, and incubation of the tissue sections with the protein A-gold complex alone [32].
A quantitative evaluation of the intensity of labelling was performed as described previously [33] for each antigen over the following renal basement membranes: the glomerular basement membrane, the extracellular matrix of the mesangial area, the epithelial basement membrane of the Bowman's capsule and that of the proximal convoluted tubule. The proximal tubule was identified according to its morphological characteristics. The quantitative evaluation was performed as follows: the tissues were first observed at low magnification at which the labelling is undiscernible. At least four glomeruli per animal were then selected randomly. For each antigen, and for each animal in both groups (four animals per group), 12 micrographs of each type of basement membrane were recorded and brought to a final magnification of $48,000 \mathrm{X}$. The intensity of labelling, evaluated as the number of gold particles per unit of area of basement membrane, was determined using a MOP-3 electronic digitizer (Carl Zeiss Inc., Québec, Canada).

In a second study, the labellings obtained for the three antigens were further analysed to determine the ultrastructural distribution of each monomer over the thickness of the glomerular basement membrane. To do so, a Bioquant image analyzer program coupled to an Apple IIe computer was used. In a first step, the distance " $\mathrm{d}$ " between each gold particle and the abluminal membrane of the endothelial cell was measured. The thickness "T" of the glomerular basement membrane at the same site was then measured. The ratio $(\mathrm{R}=\mathrm{d} / \mathrm{T})$ was calulated and the results, obtained for each antigen in each group of animals, were reported as histograms illustrating the distribution of the gold particles over the basement membrane thickness as described previously [41]. For this part of the study, an average of over one thousand gold particles was analysed for each antigen in each group.

\section{Statistical analysis}

Statistical analyses were performed between control and diabetic animals using the Student's $t$-test. Significance threshold was fixed a priori to $p<0.05$.

\section{Results}

The streptozotocin injection induced an hyperglycaemic state $24 \mathrm{~h}$ after its administration. High blood glucose levels were consistently detected in the diabetic animals throughout the entire length of the experiment which lasted 12 months. Indeed, the diabetic animals displayed higher blood glucose with glucosuria when compared to the controls. In addition, at the time of sampling, blood glucose and insulin levels for diabetic animals averaged $19.9 \pm 1.1 \mathrm{mmol} / \mathrm{l}$ and $26.7 \pm 1.2 \mathrm{mU} / \mathrm{l}$ respectively, vs $8.2 \pm 0.2 \mathrm{mmol} / \mathrm{l}$ and $76.7 \pm 16.4 \mathrm{mU} / \mathrm{l}$ for the controls. Previous studies have also shown that the hyperglycaemic state induced by streptozotocin was retained throughout the entire length of the experiment if no insulin treatment was given [42]. In addition to the hyperglycaemic state the animals developed characteristic cataract lesions and impairment of weight gain. Typical alterations of diabetic glomerulosclerosis were present in renal tissues from the hyperglycaemic animals. A thickening of the basement membranes and accumulation of mesangial matrix were observed [41, 42]. The morphometrical analysis confirmed these observations, the glomerular basement membrane thickness of the diabetic animals averaging $429.7 \pm 2.6 \mathrm{~nm}$ vs $334.2 \pm 2.6 \mathrm{~nm}$ for the age matched controls $(p<0.01)$. 

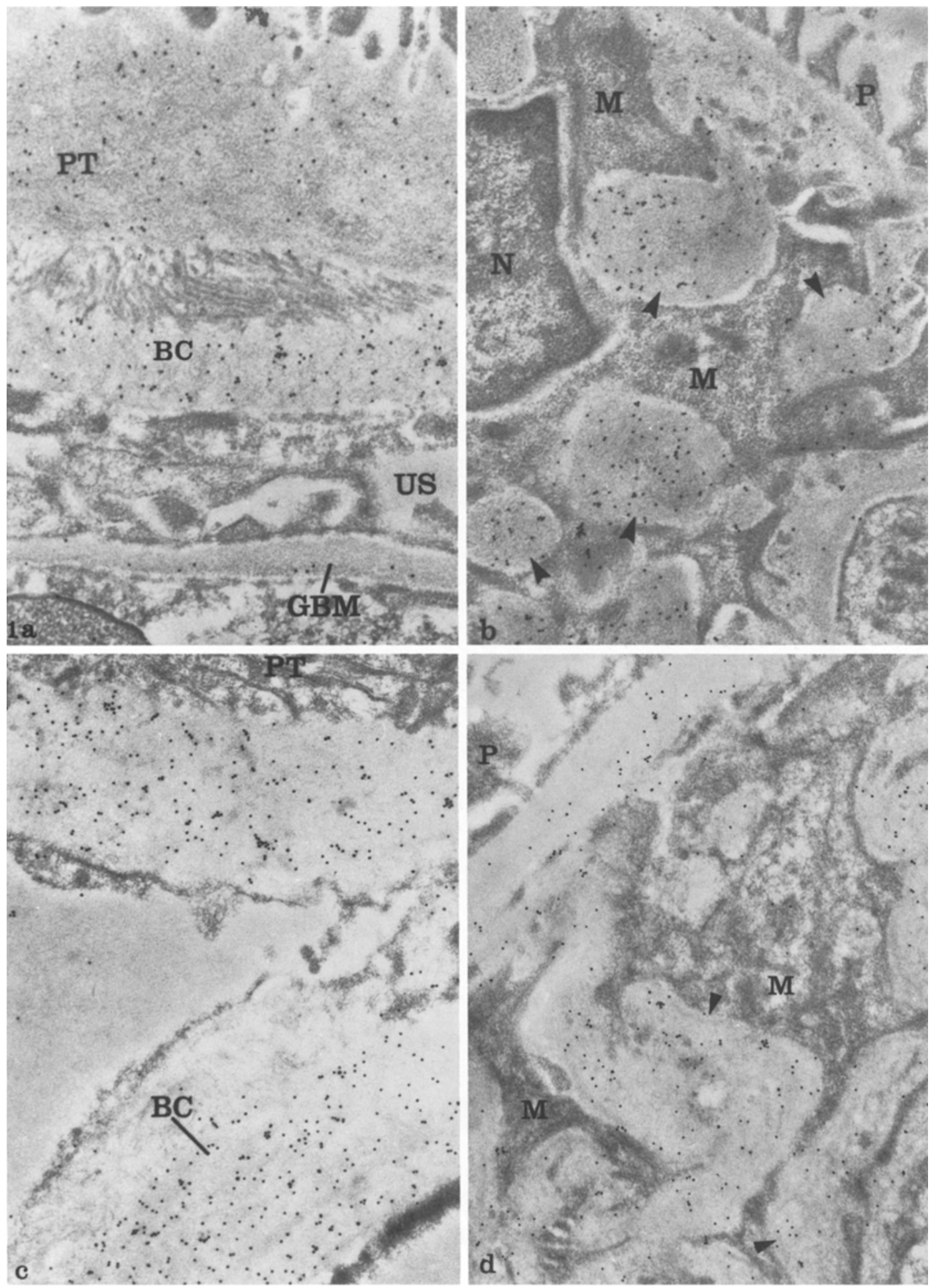

Fig. 1a-d. Localization of M1 monomers of the $\mathrm{NCl}$ domain of type IV collagen over 12 months normal and

streptozotocin-induced diabetic rat renal tissues. For the normal animal (a and $\mathbf{b}$ ), the labellings are observed in proximal tubule (PT) and

Bowman's capsule (BC) basement membranes, while the glomerular basement membrane (GBM) is labelled with lower intensity (a). The endothelial aspect of the glomerular basement membrane (a) is labelled with higher intensity than the epithelial side. In the mesangial region (b) the basement membrane underneath the podocyte $(\mathrm{P})$ is weakly labelled, while the matrix (arrow heads) is highly labelled. For the diabetic animal (c and d), the proximal tubule (PT) and

Bowman's capsule (BC) basement membranes (c) appear to be labelled with higher intensities. The mesangial matrix remains highly labelled (d). $\mathrm{N}$, nucleus of the mesangial cell; $\mathrm{M}$, mesangial cell; US, urinary space.

(a) $23,000 \times$

(b) $22,000 \times$, (c) 22,000 ,

(d) $20,000 \times$

The antigenic sites of M1, M2* and M3 monomers of the NC1 domain of type IV collagen were revealed over various renal basement membranes of the control and diabetic rats. The results are illustrated in Figures 1-4. In each case, the labellings were restricted to basement membrane material, only a few gold particles being present over other extracellular structures. In control animals, labelling of high intensities were observed for M1 monomers over the proximal tubule and the Bowman's capsule basement membranes (Fig. 1 a) as well as over the mesangial matrix (Fig.1b). These labellings appeared distributed over the entire area of the basement membranes. On the other hand, the labelling of the glomerular basement membrane was of lower intensity and present mainly on the endothelial side of the basement membrane (Figs. $1 \mathrm{a}$ and $4 \mathrm{a}$ ). In diabetic animals, the proximal tubule and the Bowman's capsule basement membranes (Fig.1c), as well as the mesangial matrix (Fig. 1d), re- 

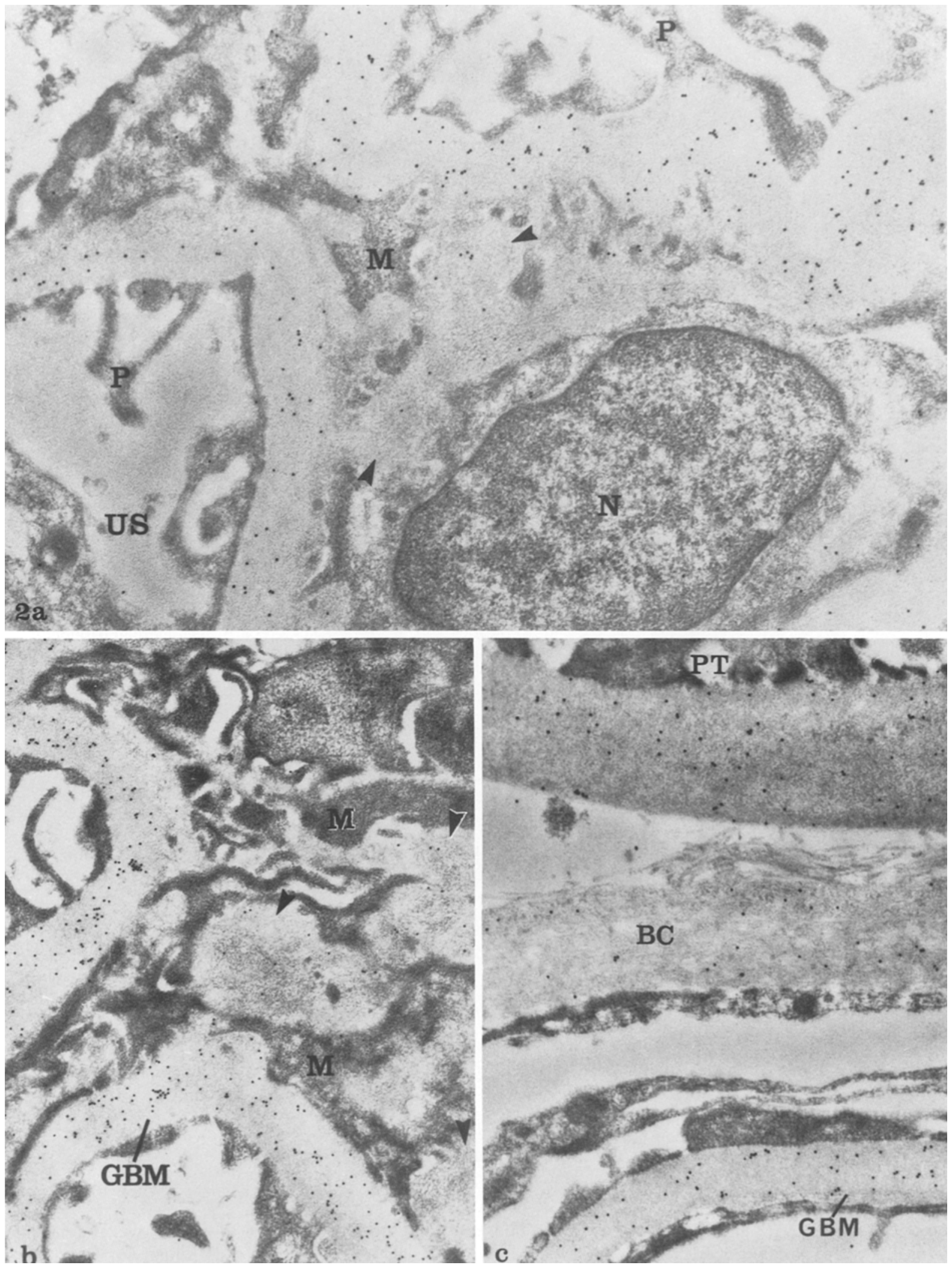

Fig. 2anc. Localization of $\mathrm{M}^{*}$ monomer of the $\mathrm{NC} 1$ domain of type IV collagen over 12 months normal and streptozotocin-induced diabetic rat renal tissues. For the normal animal (a) the labelling is observed over the basement membrane underneath the podocyte (P), while the mesangial matrix (arrow heads) is weakly labelled. For the diabetic animal (b and c), the mesangial matrix (arrow heads) is weakly labelled (b). The proximal tubule (PT) basement membrane and the glomerular basement membrane (GBM) are highly labelled, while the one of the Bowman's capsules (BC) appears labelled with lower intensity (c). The labelling in the glomerular basement membrane is present over the entire thickness. $\mathrm{N}$, nucleus of mesangial cell; $M$, mesangial cell; US, urinary space. (a) $22,000 \times$, (b) $18,000 \times$, (c) $22,000 \times$ 

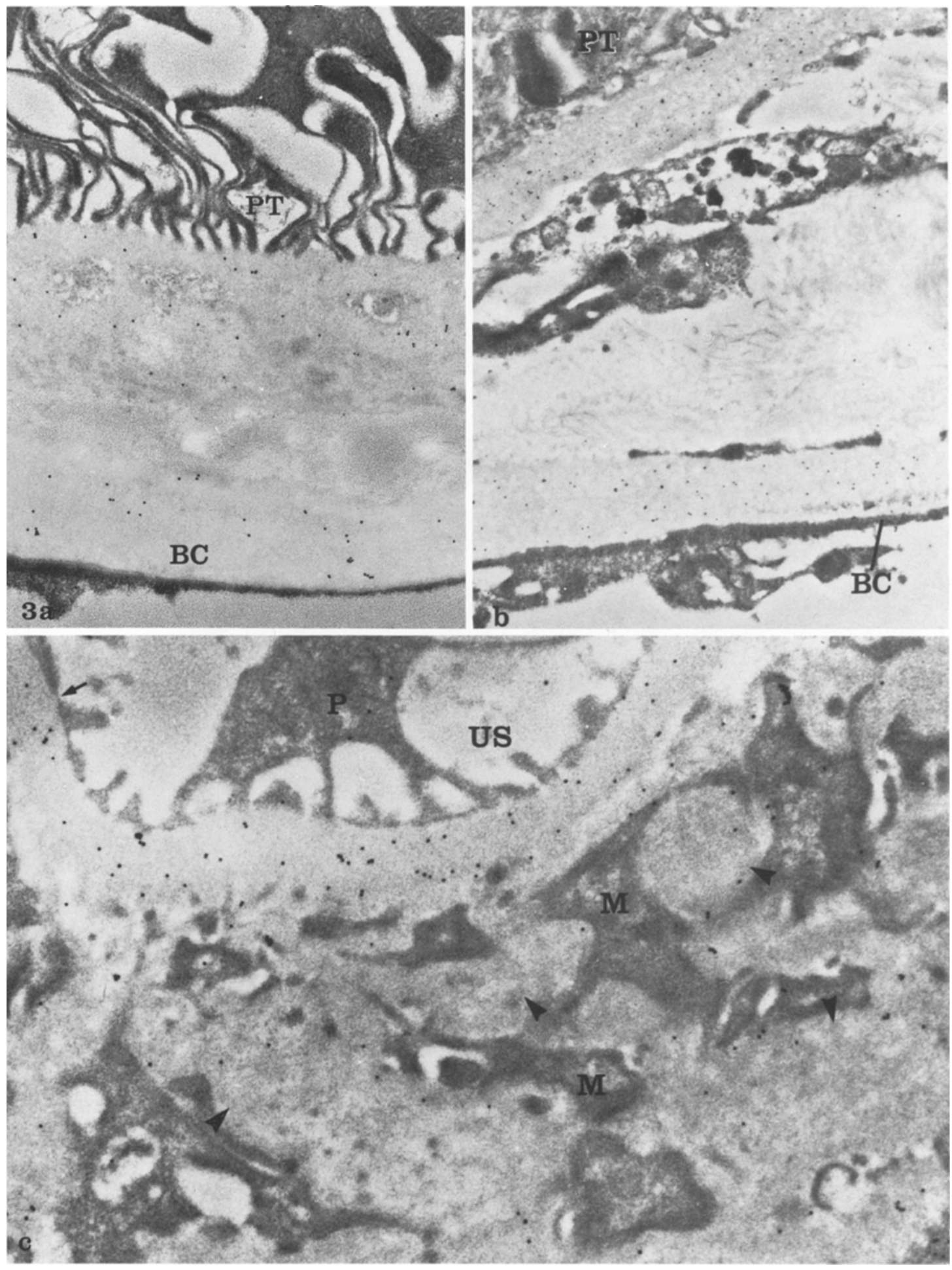

Fig. 3a-c. Localization of M3 monomer of the NC1 domain of type IV collagen over 12 months normal and streptozotocin-induced diabetic rat renal tissues. For the normal animal (a), the labellings are observed over the proximal tubule (PT) and the Bowman's capsule (BC) basement membranes. For the diabetic animal (b and $\mathbf{c}$ ), the labelling is observed over the proximal tubule (PT) and the Bowman's capsule basement membranes (BC) (b). The mesangial matrix (c) (arrow heads) is weakly labelled, while the basement membrane underneath the podocyte (P) is labelled. M, mesangial cell; US, urinary space. (a) $16,000 \times$, (b) 12,000 , (c) 24,000 mained highly labelled. The first two being more intensely labelled than in control animals. The glomerular basement membrane (Fig. 4b) also appeared to be more intensely labelled than in controls. However, and in contrast to the normal condition, the labelling for M1 was observed over the entire thickness of the glomerular basement membrane (Fig. 4b). The labellings for $\mathrm{M} 2 *$ monomer in the control animals were intense over the glomerular (Fig. $4 \mathrm{c}$ ) and the proximal tubule basement membranes. The Bowman's capsule basement membrane was labelled 


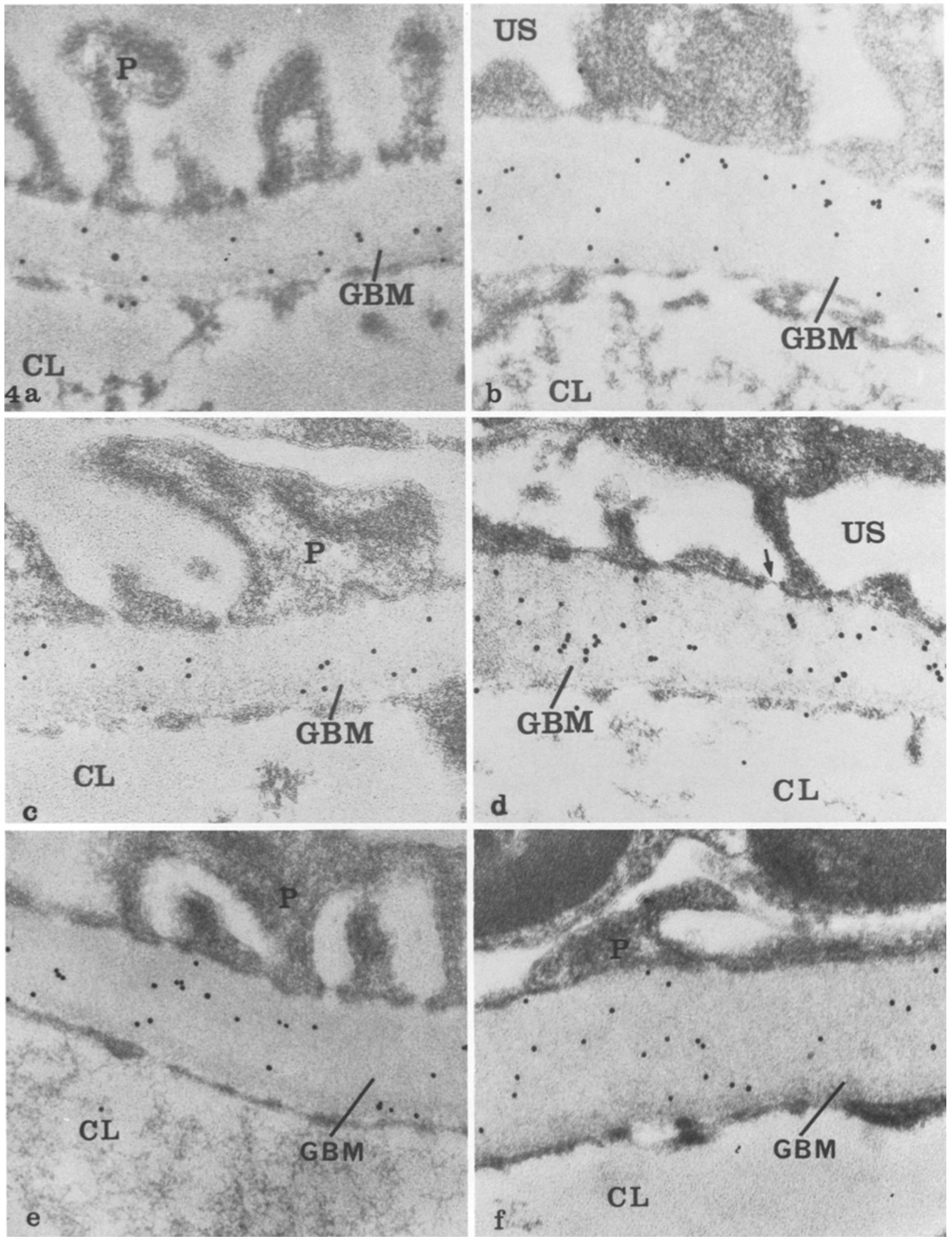

Fig. 4a-f. Localization of M1, M2* and M3 monomers over the glomerular basement membrane of 12 months normal and streptozotocin-induced diabetic rat renal tissues. a M1 control; b M1 diabetic; c M2* control; d M2* diabetic; e M3 control; f M3 diabetic; $\mathrm{CL}$, capillary lumen; GBM, glomerular basement membrane; US, urinary space. (a-f) 41,000 with lower intensity, while the mesangial matrix (Fig. 2a) displayed only few gold particles. In diabetic animals, while the mesangial matrix (Fig. 2 b) remained weakly labelled, the labelling of the proximal tubule, the Bowman's capsule and the glomerular basement membranes (Fig. 2c and $4 \mathrm{~d}$ ) appeared to be more intense than that of the controls. In all cases, the spatial distribution of $\mathrm{M} 2 *$ labellings remained similar. Labellings for M3 monomer in control animals were found over the proximal tubule, the Bow- 

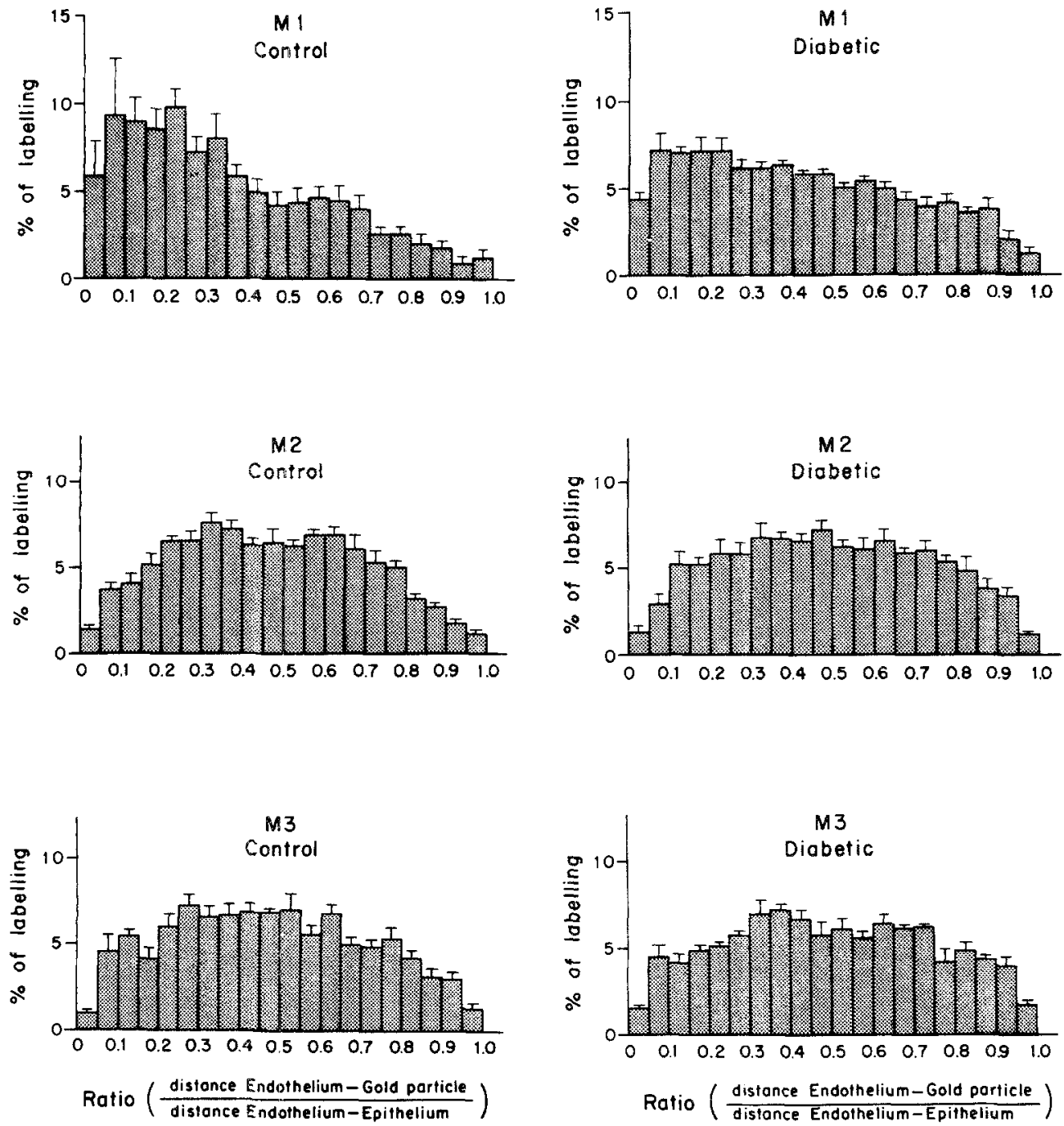

Fig.5. Histograms of the distribution of the different monomers over the average thickness of the glomerular basement membrane of control and diabetic animals. M1 monomers are asymetrically distributed in control animals. A peak of labelling is observed on the endothelial side of the basement membrane. From this peak the labelling decreases towards the epithelial cell. In long-term diabetic animals, M1 monomers are more uniformly distributed through the entire thickness of the basement membrane. M2* and M3 monomers are distributed through the entire thickness of the basement membrane with a prominent labelling of the lamina densa. No modification in the distribution of these monomers is observed in diabetic animals (over one thousand gold particles were analysed for each monomer in both groups of animals) man's capsule (Fig.3a) and the glomerular basement membranes (Fig. 4e), while the mesangial matrix was practically unlabelled. No major difference was observed in the diabetic animals; the proximal tubule and the Bowman's capsule basement membranes (Fig. 3b), as well as the glomerular basement membranes (Fig. 4f) were labelled, while the mesangial matrix (Fig. 3c) remained very weakly labelled.

A quantitative analysis of the intensity of labelling for each monomer in the various renal basement membranes was performed. The results, expressed as number of gold particles per $\mu \mathrm{m}^{2}$ of basement membrane as well as their statistical analysis, are reported in Table 1 . They indicate that the different basement membranes studied are heterogeneous in terms of their respective labelling for each antigen. This was found to be the case in both control and diabetic animals. Furthermore, they reveal an important increase in intensities of the labelling for M1 and M2* monomers during diabetes. This increase was observed in all basement membranes except for the mesangial matrix, the labelling of which was unchanged during diabetes (Table 1). Conversely, for M3 monomer, no difference in intensities of labelling was found between basement membranes of control and diabetic animals.
The labelling over the glomerular basement membrane was further analysed to determine the spatial distribution of M1, M2* and M3 monomers through the thickness of the basement membrane. The results presented as histograms are shown in Figure 5. In control animals, labelling for M1 monomers is preferentially distributed on the endothelial side of the basement membrane where a peak of labelling is observed. From this peak, the labelling decreases towards the epithelial side. In contrast, in diabetic animals the labelling is more uniformly distributed through the entire thickened basement membrane. Labellings for M2* and M3 monomers in control and in diabetic animals are found to be distributed over the entire thickness of the lamina densa, few gold particles being present over the laminae rarae.

The specificity of the results as well as the absence of major cross reactivity between the antibodies were demonstrated by immunoblot analysis $[28,40]$ and by our labelling experiments. Indeed, incubation of tissue sections with a normal rabbit serum prior to the protein A-gold step resulted in very low labelling, only scattered gold particles were seen on the tissue. This demonstrates absence of nonspecific interactions of immunoglobulins to the tissue sections. Incubation with the protein A-gold alone also resulted in an absence of labelling (results not shown). 
Table 1. Intensities of the labellings for M1, M2* and M3 monomers over various renal basement membranes (gold particles/ $\mu m^{2}$ of basement membrane)

\begin{tabular}{llllll}
\hline & & GBM & MM & BC & PT \\
\hline M1 & Control & $17.88 \pm 4.64$ & $36.94 \pm 8.78$ & $46.63 \pm 10.74$ & $31.89 \pm 9.43$ \\
& Diabetic & $34.36 \pm 3.73^{\mathrm{b}}$ & $32.70 \pm 3.81$ & $82.52 \pm 12.18^{\mathrm{b}}$ & $47.67 \pm 5.70$ \\
M2* & Control & $36.93 \pm 4.60$ & $7.91 \pm 1.35$ & $14.72 \pm 2.80$ & $22.10 \pm 4.19$ \\
& Diabetic & $54.53 \pm 5.07^{\mathrm{b}}$ & $9.73 \pm 2.01$ & $29.96 \pm 2.48^{\mathrm{a}}$ & $36.11 \pm 1.20^{\mathrm{b}}$ \\
M3 & Control & $30.65 \pm 2.65$ & $7.48 \pm 0.56$ & $12.13 \pm 0.66$ & $16.66 \pm 2.37$ \\
& Diabetic & $32.71 \pm 1.44$ & $5.60 \pm 0.13$ & $15.18 \pm 1.04$ & $16.92 \pm 1.55$ \\
\hline
\end{tabular}

mean $\pm \operatorname{SEM}(n=4)$

GBM: glomerular basement membrane; MM: mesangial matrix; BC: Bowman's capsule basement membrane; PT: proximal tubule basement membrane

${ }^{\mathrm{a}} p<0.01 ;{ }^{\mathrm{b}} p<0.05$

\section{Discussion}

The streptozotocin-injected animals displayed metabolic changes related to diabetes, namely high blood glucose, low insulin levels, proteinuria and deficiency in weight gain as reported previously $[41,42]$. They also displayed the typical kidney alterations described in experimental diabetes [43] with significant thickening of the glomerular basement membrane and accumulation of mesangial matrix $[41,42]$. A relative thickening of the glomerular basement membrane was also observed with aging in control animals which confirms previous results $[42,43]$. The absence of direct effect of streptozotocin on the development of these alterations was demonstrated previously $[44,45]$.

Studies performed on the NC1 domain of type IV collagen from bovine glomerular basement membrane led to the conclusion that two new collagenous chains designated $\alpha 3$ (IV) and $\alpha 4$ (IV) do exist beside the $\alpha 1$ (IV) and $\alpha 2$ (IV) chains $[28,29]$. Immunocytochemical studies have previously demonstrated that these chains are not codistributed in normal renal basement membranes [30-32] nor in human diabetic glomerular basement membrane [34]. On the other hand, in diabetic conditions the distribution of type IV collagen in glomerular basement membrane was found to be altered [20]. These data prompted us to further investigate at the ultrastructural level, possible changes in the distribution of $\mathrm{M} 1, \mathrm{M} 2 *$ and M3 monomers in renal basement membrane of diabetic rats. To do so, we used specific polyclonal antibodies and applied the protein A-gold immunocytochemical technique to reveal with high resolution these monomers in renal basement membranes of control and long-term diabetic rats. The heterogeneous distribution of M1, M2* and M3 monomers described previously in renal basement membranes of 3-months-old normoglycaemic rats [32] was confirmed in the present study on renal tissue of 12-months-old control animals. In the diabetic condition however, we found changes in the distribution of the monomers and in the intensity of their immunolabellings. These modifications varied according to the monomer analysed as well as to the basement membrane considered. Indeed, a 1.5 to 2 -fold increase in the labelling intensities of M1 and M2* monomers was observed for proximal tubule and Bowman's capsule basement membranes of diabetic animals, while the intensity of M3 labelling remained unchanged. The labelling for each monomer appeared equally distributed over the entire area of these basement membranes in control and diabetic animals. On the other hand, evolution of glomerular basement membrane and mesangial matrix immunolabellings in glomeruli from diabetic rats was quite distinct. The labelling intensities of $\mathrm{M} 1$ and $\mathrm{M} 2 *$ monomers were increased within the glomerular basement membrane but remained unchanged in the mesangial matrix. Furthermore, the ultrastructural distribution of M1 monomers was altered. It was present through the entire thickness of the basement membrane in diabetic animals whereas it predominated on the endothelial side in controls. For the M2* monomer, only an increase in its labelling intensity was observed, the distribution remaining the same. Finally, for the M3 monomer neither its labelling nor its distribution were modified during diabetes.

Our results, showing an overall increase in the labelling intensities of M1 and M2 monomers in glomerular basement membrane, could be related to an increase in their content which correlates with the biochemical demonstration of an accumulation of type IV collagen in basement membranes during diabetes $[16,17,46]$. However, new information is provided by our quantitative study demonstrating that the $\alpha 1$ (IV) and $\alpha 2$ (IV) chains, recognized by the anti-M1 antibodies, and the $\alpha 3$ (IV) chain, recognized by the anti $\mathrm{M} 2 *$ antibody, participate in the accumulation of collagenous material in diabetic renal basement membranes. Surprisingly, the $\alpha 4$ (IV) chain appears not to be involved in this process. Concerning basement membranes of the glomeruli, an unexpected finding is the striking difference observed between the mesangial matrix and the peripheral basement membrane. Expansion of the matrix within the mesangial area is a distinctive and constant lesion in diabetic glomerulosclerosis. This, together with the accumulation of type IV collagen extracted from diabetic glomeruli, suggests that this accumulation would take place in mesangial matrix. Our study has, however, clearly demonstrated that the increased concentration of $\alpha 1(\mathrm{IV})$ and $\alpha 2$ (IV) chains of type IV collagen does not occur in the mesangial matrix but rather in the peripheral glomerular basement membrane. The discrepency between the stability of the various chains of the mesangial type IV collagen and their imbalance in the peripheral basement membrane could not have been detected by biochemical 
methods where the mesangial matrix and the peripheral basement membranes are extracted and analysed as a whole. These results show that the glomerular basement membrane and the extracellular matrix of the mesangial area differ not only in their collagen composition but also in their response to the metabolic disturbances induced by diabetes.

In renal basement membranes from diabetic animals, the absence of detectable modifications in M3 monomer in contrast to the marked increase in $\mathrm{M} 2 *$ monomer is interesting to consider. These monomers have been previously shown to be codistributed within renal basement membranes in normal conditions [32]. In addition, the absence of M2* monomer in patients affected with Alport's syndrome has also been correlated with an absence of the M3 monomer (unpublished personal data). This codistribution of $\mathrm{M} 2 *$ and $\mathrm{M} 3$ monomers could suggest that the $\alpha 3(\mathrm{IV})$ and $\alpha 4(\mathrm{IV})$ chains belong to the same molecule. However, our data in the diabetic rats where only the M2* monomer is increased, suggest that $\alpha 3$ (IV) and $\alpha 4$ (IV) chains may in fact belong to different molecules. Another hypothesis could be that the molecular arrangement of these collagen chains is modified during diabetes. To what extent modification of the basement membrane morphological and structural integrity alter their functional properties is under investigation. The study should not be limited to type IV collagen since changes in the non-collagenous components of the basement membrane such as laminin and heparan sulfate proteoglycan have also been reported during diabetes [19, $47,48]$. Non-enzymatic glycosylation also affects basement membrane components [49-52] and their binding properties [53]. All these results suggest that an important reorganisation of the structure of basement membranes must take place during diabetes as a consequence of the unbalanced content of their various components and their biochemical modifications.

Our study thus demonstrates alterations in the type IV collagen composition of basement membranes during diabetes. These alterations are characterized by an increase in $\mathrm{M} 1$ and $\mathrm{M} 2 *$ monomers, corresponding to the $\alpha 1$ (IV), $\alpha 2$ (IV) and $\alpha 3$ (IV) chains of type IV collagen, in all renal basement membrane studied except in the mesangial matrix. The M3 monomer, corresponding to the $\alpha 4(\mathrm{IV})$ chain was, however, not altered. In addition, modifications in the spatial distribution of M1 monomers were observed in the glomerular basement membrane. These results demonstrate that the collagenous nature of some basement membranes are significantly modified during diabetes. The importance of these modifications suggests that a rearrangement of the structural organization of basement membranes and particularly of the glomerular basement membrane occurs, possibly leading to alterations in their functional properties.

Acknowledgements. We are grateful to Ms. D.Gingras, Ms. C. Venne and Ms. J. Leveillé for their excellent technical assistance, and to Ms. J.Cainey for secretarial work. This study was supported by grants from the Medical Research Council of Canada and la Faculté de Médecine Necker Enfants-Malades, Université de Paris V. MB and MD are recipients of a Scientist and a Studentship Awards from the Medical Research Council of Canada respectively.

\section{References}

1. Kimmelstiel P, Wilson C (1936) Intercapillary lesions in glomeruli of kidney. Am J Pathol 12:83-89

2. Farquhar MG, Hopper J Jr, Moon HD (1959) Diabetic glomerulosclerosis: electron and light microscopic studies. Am J Pathol 35: 721-753

3. Lazarow A (1967) Glomerular basement membrane thickening in diabetes. In: Ostman J, Milner RD (eds) Proc. of the 6th Inter. Cong. Diab. Fed. Excerpta Medica, Amsterdam, pp 301306

4. Østerby R (1975) Early phases in the development of diabetic glomerulopathy: a quantitative electron microscopic study. Acta Med Scand [Suppl 574]: 1-82

5. Mogensen CE, Østerby R, Gundersen HJG (1979) Early fonctional and morphologic vascular renal consequences of the diabetic state. Diabetologia 17: 71-76

6. Viberti $G$, Keen $H$ (1984) The patterns of proteinuria in diabetes mellitus. Relevance to pathogenesis and prevention of diabetic nephropathy. Diabetes 33: 686-692

7. Viberti G, MacKintosh D, Bilous RW, Pickup JC, Keen H (1982) Proteinuria in diabetes mellitus: role of spontaneous and experimental variation of glycemia. Kidney Int 21: 714-720

8. Mauer MS, Steffes MW, Ellis EN, Sutherland DER, Brown DM, Goetz FC (1984) Structural-functional relationships in diabetic nephropathy. J Clin Invest 74: 1143-1155

9. Beisswenger PJ, Spiro RG (1973) Studies in the human glomerular basement membrane: composition, nature of the carbohydrate units and chemical changes in diabetes mellitus. Diabetes 22: 180-193

10. Cruz A, Moreau-Lalande H (1978) Biochemical studies on glomerular basement membrane in human diabetic microangiopathy. Pathol Biol 26: 411-417

11. Kefalides NA (1974) Biochemical properties of human glomerular basement membrane in normal and diabetic kidneys. J Clin Invest 53: 403-407

12. Spiro RG (1976) Search for a biochemical basis of diabetic microangiopathy. Diabetologia 12: 1-14

13. Wahl P, Deppermann D, Hasslacher C (1982) Biochemistry of glomerular basement membrane of the normal and diabetic human. Kidney Int 21: 744-749

14. Westberg GW, Michael AF (1973) Human glomerular basement membrane: Chemical composition in diabetes mellitus. Acta Med Scand 194: 39-47

15. Bendayan M, Benhamou N, Desjardins M (1990) Ultrastructural distribution of lectin-binding sites in the glomerular wall of streptozotocin-induced diabetic rats. J Submicrosc Cytol Pathol 22: 173-184

16. Brownlee M, Spiro RG (1979) Glomerular basement membrane metabolism in the diabetic rat. In vivo studies. Diabetes 28: 121-125

17. Karttunen T, Risteli J, Autio-Harmainen H, Risteli L (1986) Effect of age and diabetes on type IV collagen and laminin in human kidney cortex. Kidney Int 30: 586-591

18. Parthasarathy N, Spiro RG (1982) Effect of diabetes on the glycosaminoglycan component of the human glomerular basement membrane. Diabetes 31: 738--741

19. Rohrbach DH, Hassell JR, Kleinman HK, Martin GR (1982) Alterations in the basement membrane (heparan sulfate) proteoglycan in diabetic mice. Diabetes $31: 185-188$

20. Bendayan M (1985) Alteration in the distribution of type IV collagen in glomerular basal laminae in diabetic rats as revealed by immunocytochemistry and morphological approach. Diabetologia 28: $373-378$

21. Leblond CP, Inoue S (1989) Structure, composition, and assembly of basement membrane. Am J Anat 185: 367-390

22. Timpl R, Wiedemann $H$, Delden V van, Furthmayr $H$, Kühn K (1981) A network model for the organization of type IV collagen molecules in basement membranes. Eur J Biochem 120: 203-211 
23. Tsilibary EC, Charonis AS (1986) The role of the main noncollagenous domain (NC1) in type IV collagen self-assembly. J Cell Biol 103: 2467-2473

24. Weber S, Dolz R, Timpl R, Fessler JH, Engel J (1988) Reductive cleavage and reformation of the interchain disulfide bonds in the globular hexameric domain $\mathrm{NC1}$ involved in network assembly of basement membrane collagen (type IV). Eur J Biochem 175: 229-236

25. Yurchenco PD, Furthmayr H (1984) Self-assembly of basement membrane collagen. Biochemistry 23: 1839-1850

26. Yurchenco PD, Tsilibary EC, Charonis AS, Furthmayr H (1986) Models for the self-assembly of basement membrane. J Histochem Cytochem 34: 93-102

27. Yurchenco P, Ruben GC (1987) Basement membrane structure in situ: Evidence for lateral associations in type IV collagen network. J Cell Biol 105: 2559-2568

28. Butkowski RJ, Wieslander J, Wisdom BJ, Barr JF, Noelken ME, Hudson BG (1985) Properties of the globular domain of type IV collagen and its relationship to the Goodpasture antigen. J Biol Chem 260: 3739-3747

29. Butkowski RJ, Langeveld JPM, Wieslander J, Hamilton J, Hudson BG (1987) Localization of the Goodpasture epitope to a novel chain of basement membrane collagen. J Biol Chem 262: 7874-7877

30. Butkowski RJ, Wieslander J, Kleppel M, Michael AF, Fish AJ (1989) Basement membrane collagen in the kidney: regional localization of novel chains related to collagen IV. Kidney Int 35: 1195-1202

31. Mounier F, Gros F, Wieslander J, Hinglais N, Sich M, Guicharnaud L, Gubler MC (1988) Glomerular distribution of M1 and M2 subunits of the globular domain of the basement membrane collagen. An immunohistochemical study. In: Gubler MC, Sternberg M (eds) Progress in basement membrane research. Renal and related aspect in health and disease. John Libbey Eurotext, London, pp 53-59

32. Desjardins M, Gros F, Wieslander J, Gubler MC, Bendayan M (in press) Heterogeneous distribution of monomeric elements from the globular domain ( $\mathrm{NC1}$ ) of type IV collagen in renal basement membranes as revealed by high resolution quantitative immunocytochemistry. Lab Invest

33. Desjardins M, Bendayan M (1989) Heterogenous distribution of type IV collagen, entactin, heparan sulfate proteoglycan and laminin among renal basement membranes as demonstrated by quantitative immunocytochemistry. J Histochem Cytochem 37 : 885-897

34. Gubler MC, Noël LH, Mounier F, Gros F, Wieslander J (1988) Immunohistochemical study of extracellular matrix components in diabetic glomerulosclerosis. In: Gubler MC, Sternberg M (eds) Progress in basement membrane research. Renal and related aspects in health and disease. John Libbey Eurotext, London, pp 201-204

35. McLean IW, Nakane PK (1974) Periodate-lysine-paraformaldehyde fixative. A new fixative for immunoelectron microscopy. $\mathrm{J}$ Histochem Cytochem 22: 1077-1083

36. Bendayan M (1984) Protein A-gold electron microscopic immunocytochemistry; methods, applications and limitations. J Elec Micr Tech 1: 243-270

37. Hudson BG, Wieslander J, Wisdom BJ Jr, Noelken ME (1989) Goodpasture syndrome: molecular architecture and function of basement membrane antigen. Lab Invest 61:256-269

38. Butkowski RJ, Shen GQ, Wieslander J, Michael AF, Fish AJ (1990) Characterization of type IV collagen $\mathrm{NC}_{1}$ monomers and goodpasture antigen in human renal basement membranes. $\mathrm{J}$ Lab Clin Med 115: 365-373
39. Gunman S, Saus J, Noelken ME, Hudson BG (1990) Glomerular basement membrane. Identification of a fourth chain, $\alpha \mathrm{A}$, of type IV collagen. J Biol Chem 265: 5466-5469

40. Langeveld JPM, Wieslander J, Timoneda J, McKinney P, Butkowski RJ, Wisdom BJ Jr, Hudson BG (1988) Structural heterogeneity of the noncollagenous domain of basement membrane collagen. J Biol Chem 263: 10481-10488

41. Bendayan M, Gingras D, Charest P (1986) Distribution of endogenous albumin in the glomerular wall of streptozotocin-induced diabetic rats as revealed by high resolution immunocytochemistry. Diabetologia 29: 868-875

42. Desjardins M, Bendayan M (1989) Ultrastructural distribution of endogenous IgGs in the glomerular wall of control and diabetic rats. Histochem $\mathrm{J} 21: 731-742$

43. Hirose K, Østerby R, Nozawa M, Gundersen HJG (1982) Development of glomerular lesions in experimental long-term diabetes in the rat. Kidney Int 21: 689-695

44. Rasch R (1979) Prevention of diabetic glomerulopathy in streptozotocin diabetic rats by insulin treatment. Mesangial region. Diabetologia 17: 243-248

45. Rasch R (1979) Prevention of diabetic glomerulopathy in streptozotocin diabetic rats by insulin treatment. Albumin excretion. Diabetologia 18: 413-416

46. Grant ME, Harwood R, Williams IF (1976) Increased synthesis of glomerular basement membrane collagen in streptozotocin diabetes. J Physiol (London) 257: 56P-57P

47. Kanwar YS, Rosenzweig LJ, Linker A, Jakubowski ML (1983) Decreased de novo synthesis of glomerular proteoglycans in diabetes: Biochemical and autoradiographic evidence. Proc Natl Acad Sci USA 80: 2272-2275

48. Shimomura H, Spiro RG (1987) Studies on macromolecular components of human glomerular basement membrane and alterations in diabetes. Decreased levels of heparan sulfate proteoglycan and laminin. Diabetes 36: $374-381$

49. Cohen MP, Urdanivia E, Surma M, Van-Yu W (1980) Increased glycosylation of glomerular basement membrane collagen in diabetes. Biochem Biophys Res Comm 95: 765-769

50. Garlick RL, Bunn HF, Spiro RG (1988) Nonenzymatic glycation of basement membranes from human glomeruli and bovine sources. Effect of diabetes and age. Diabetes 37: 1144-1150

51. Monnier VM, Kohn RR, Cerami A (1984) Accelerated age-related browning of human collagen in diabetes mellitus. Proc Natl Acad Sci USA 81: 583-587

52. Vlassara H, Brownlee M, Cerami A (1986) Nonenzymatic glycosylation: role in the pathogenesis of diabetic complications. Clin Chem 32 (B): B37-B41

53. Tarsio JF, Reger LA, Furcht LT (1988) Molecular mechanisms in basement membrane complications of diabetes. Alterations in heparin, laminin, and type IV collagen association. Diabetes 37 : 532-539

Received: 6 April 1990

and in revised form: 24 July 1990

Dr. M. Bendayan

Département d'Anatomie

Faculté de Médecine

Université de Montréal

C. P. 6128, Succursale A

Montréal, Québec H3C 3J7

Canada 